\title{
EVALUACIÓN FORMATIVA EN LA INSTRUMENTACIÓN DE CANCIONES. UNA BUENA PRÁCTICA EN EL CONSERVATORIO
}

Formative assessment in the instrumentation of songs. A good practice at the conservatory

Avaliação formativa na instrumentação de canções. Uma boa prática no conservatório

\section{Inés María Monreal Guerrero (1)}

\section{Elena Berrón Ruiz (2)}

(1) Universidad de Valladolid, España. Teléfono: +34 615208622. Correo electrónico: ines.monreal@mpc.ucva.es

(2) Conservatorio Profesional de Música de Segovia, España. Teléfono: +34 630589917. Correo electrónico: eberron@educa.jcyl.es

\section{Resumen}

Se presenta una buena práctica de evaluación formativa llevada a cabo en el Conservatorio Profesional de Música de Segovia con alumnos de Lenguaje Musical que cursaban el primer curso de las Enseñanzas Profesionales. Es una experiencia en la que los alumnos tienen que crear instrumentaciones para distintas canciones del ámbito del Pop-Rock, sometiéndose a una evaluación formativa por parte de la profesora, en primer lugar, y de sus propios compañeros, en un segundo momento. Los resultados han sido muy positivos, ya que, al utilizar la evaluación con el fin de ayudarles a mejorar y no como proceso para asignarles una calificación, ha aumentado su motivación hacia el aprendizaje y su satisfacción por el trabajo bien hecho, al mismo tiempo que se ha conseguido favorecer la adquisición de aprendizajes significativos.

Palabras clave: Instrumentación; evaluación formativa; aprendizaje significativo

\section{Abstract}

A good practice of formative assessment carried out in the Professional Conservatory of Music of Segovia with students of Musical Language that were studying the first course of the Professional Teachings is presented. It is an experience in which the students have to create instrumentations for different songs of the Pop-Rock field, undergoing a formative assessment from the teacher, in the first place, and from their partners lately, in a second moment. The results have been very positive, since, by using the assessment 
with the purpose of helping them to improve and not as a process to assign them a grade, it has increased their motivation towards learning and their satisfaction for a job well done, at the same time that it has been possible to favour the acquisition of significant learning.

Keywords: Instrumentation; formative assessment; meaningful learning

\section{Resumo}

Apresenta-se uma boa prática de avaliação formativa realizada no Conservatório Profissional de Música de Segóvia com estudantes de Língua Musical que estavam estudando no primeiro curso do Ensino Profissional. É uma experiência em que os alunos têm que criar instrumentação para diferentes canções do âmbito do Pop-Rock, submetendo-se, em primeiro lugar, a uma avaliação formativa por parte da professorae, num segundo momento, dos seus próprios companheiros. Os resultados foram muito positivos, uma vez que, ao utilizar a avaliação com o objectivo de os ajudar a melhorar e não como o fim de lhes atribuir uma classificação, aumentou a sua motivação para a aprendizagem e a sua satisfação por um trabalho bem feito, ao mesmo tempo que foi possível favorecer a aquisição de aprendizagens significativas.

Palavras-chave: Instrumentação; avaliação formativa; aprendizagem significativa

\section{Introducción}

Somos conocedores de la importancia de implementar, de manera eficaz, la evaluación formativa en los distintos niveles educativos. Autores como Fraile (2012), Fraile, López-Pastor, Castejón y Romero (2013), y Hortigüela, Palacios y López-Pastor (2018), entre otros, avalan la utilización de la evaluación formativa como medio de evaluación eficaz para fomentar, además de buenos resultados en los alumnos, procesos cognitivos de reflexión sobre el propio proceso de aprendizaje (Arribas, Carabias, \& Monreal, 2010).

En el ámbito de las enseñanzas artísticas, apenas existen investigaciones que tengan como objeto de estudio la evaluación formativa en los conservatorios. No obstante, destacamos la tesis doctoral de Mitchell (2012), donde se aplican procesos de evaluación cercanos a la esencia de la evaluación formativa. 
En la experiencia que presentamos, nos acercaremos a dos estrategias evaluativas: el feed-back aportado por la docente, como facilitador del aprendizaje, y la coevaluación, útil para llevar a cabo una evaluación compartida y más enriquecedora (López-Pastor, González, \& Barba, 2005). Ambas estrategias nos aportan información para saber cómo ayudar al alumno a mejorar y aprender más (López-Pastor, 2012).

\section{Contextualización}

El trabajo se ha llevado a cabo en el Conservatorio Profesional de Música de Segovia, con 34 alumnos pertenecientes a cuatro grupos de Lenguaje Musical del primer curso de las Enseñanzas Profesionales.

\section{Diseño y desarrollo}

En los distintos grupos de Lenguaje Musical, se han realizado actividades de instrumentación creativa de canciones procedentes del ámbito del Pop-Rock. Cada mes se seleccionaba un fragmento de una canción (normalmente el estribillo) y los alumnos debían transcribirlo musicalmente de oído. Después, en casa y en el plazo de una semana, los alumnos debían componer sus propias instrumentaciones, utilizando el programa informático MuseScore. Para ello, debían utilizar patrones de acompañamiento rítmico-melódicos, bajos y percusión, eligiendo los instrumentos que consideraran más convenientes (Berrón, 2017).

Una vez que daban por terminada su instrumentación, se entregaba a la profesora para su feed-back, quien les indicaba si era correcta desde el punto de vista musical o si tenían que realizar alguna modificación; con dicha retroalimentación, se favorecía su proceso de aprendizaje.

Posteriormente, cada alumno exponía su trabajo en clase, comentando la partitura y poniendo el audio resultante. En ese momento, a través de la coevaluación, el resto de los compañeros realizaba aportaciones de mejora sobre la instrumentación, las cuales quedaban registradas y enriquecían el proceso evaluativo. Los criterios utilizados en dicha coevaluación eran tres: diversidad tímbrica de los instrumentos escogidos, complementariedad de los acompañamientos realizados por cada uno de ellos y belleza musical de la composición resultante.

De todas las instrumentaciones realizadas cada mes, los alumnos elegían la que más les gustaba y se trabajaba en clase para llevarla a escena a finales de cada trimestre. 


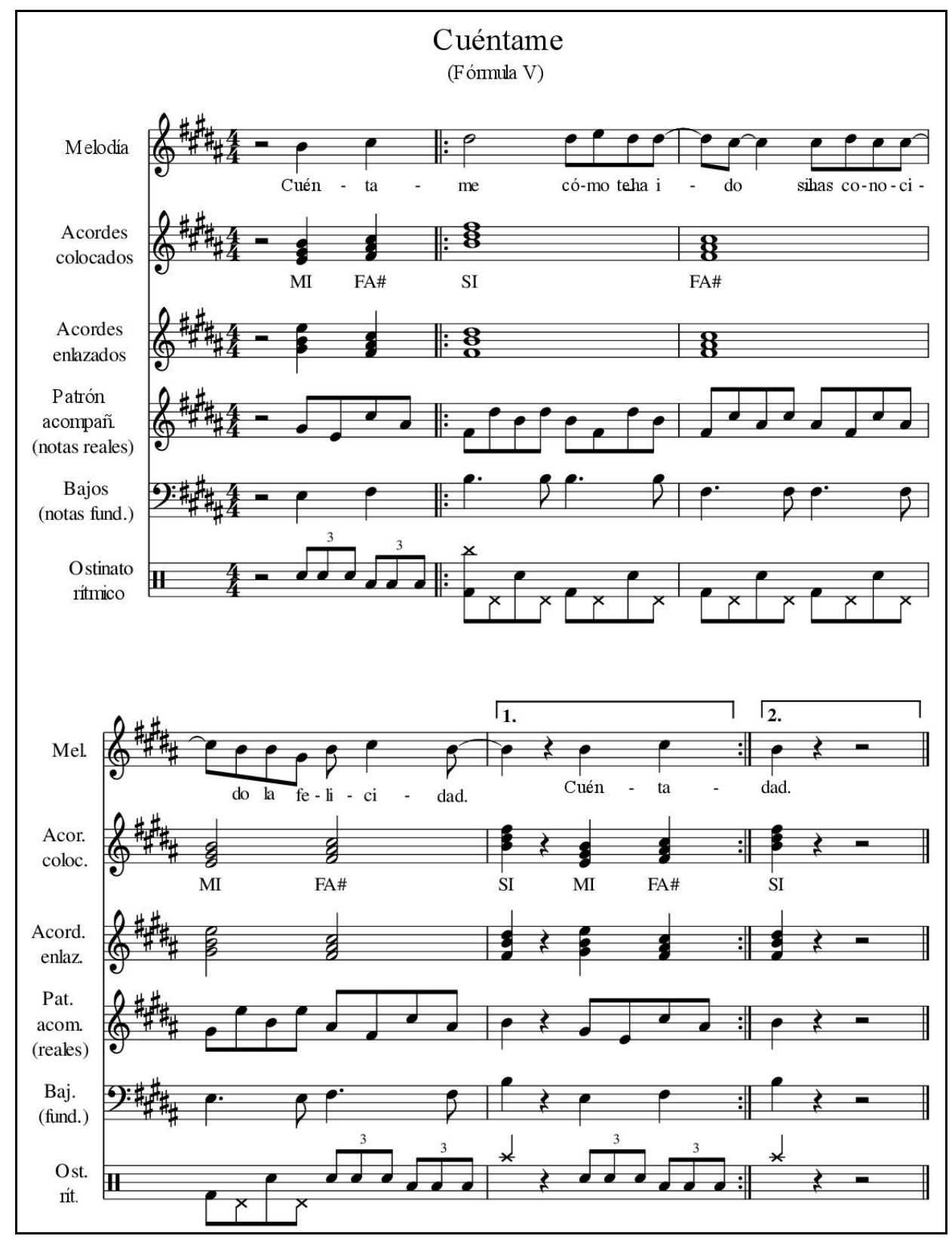

Figura 1. Instrumentación del estribillo de la canción "Cuéntame" de Fórmula V realizada por un alumno con el programa MuseScore (Berrón, 2017).

\section{Evaluación}

Las instrumentaciones realizadas por cada alumno eran sometidas a un proceso de evaluación formativa en dos momentos distintos:

1. Tras realizar en casa el trabajo descrito en el apartado anterior y dar por terminada su instrumentación, los alumnos se la entregaban a la profesora para que les diera el feed-back. Para ello, la profesora señalaba en la propia partitura 
las notas musicales que no eran correctas con color rojo y hacía comentarios al margen sobre cuáles eran las causas de los errores y cómo se podrían corregir.

2. Una vez que la instrumentación ya estaba bien construida desde el punto de vista técnico y se presentaba en clase, cada alumno recibía feed-back de sus compañeros, quienes hacían nuevas sugerencias con el fin de mejorar su calidad artística desde una perspectiva estético-musical. En este caso, las valoraciones se proporcionaban en base a los tres criterios señalados en el apartado anterior y también resultaban muy enriquecedoras para el proceso de aprendizaje.

Igualmente, cabe destacar que la votación realizada al final de cada trimestre para elegir las mejores instrumentaciones y llevarlas a escena también suponía, en cierto sentido, una forma de evaluación que motivaba a los alumnos para que se esforzaran, con más empeño, en sus creaciones.

La calificación de la asignatura se ofrece segmentada en 4 apartados: ritmo, entonación, teoría y dictado. La realización de instrumentaciones no se corresponde directamente con ninguno de ellos, por eso no se califica, pero hemos comprobado que ha contribuido a que los alumnos mejoren especialmente en teoría y dictado, al ayudarles a comprender todo el entramado musical en su conjunto y a ejercitar su capacidad auditiva, lo cual manifestaron ellos mismos en un cuestionario que les entregamos a final de curso.

La mayor dificultad que encontramos en dicha experiencia fue poder dedicar el tiempo necesario, ya que disponíamos tan solo de 2 horas semanales de clase e implicó tener que restarlo de otras actividades, pero consideramos muy beneficioso darle prioridad.

\section{Conclusiones}

Los procesos de evaluación formativa que hemos puesto en práctica en los trabajos de instrumentación han desarrollado las competencias profesionales de los alumnos (López-Pastor, 2010), al ayudarles a entender mejor los contenidos de la asignatura de forma globalizada y al favorecer la adquisición de aprendizajes significativos. Además, el alumnado ha mostrado un alto grado de satisfacción con la utilización de estos sistemas de evaluación (Romero-Martín, Castejón-Oliva, \& López-Pastor, 2015).

El hecho de utilizar la evaluación con una finalidad formativa y sin la intención de obtener una calificación determinante en su expediente les ha motivado para que realizaran las instrumentaciones con dedicación y sin escatimar esfuerzos, buscando 
como única "recompensa" la satisfacción que produce el trabajo bien hecho. Esta es, sin duda, la mayor aportación de la experiencia que hemos presentado.

\section{Referencias}

Arribas, J. M., Carabias, D., \& Monreal, I. (2010). La docencia universitaria en la formación inicial del profesorado. El caso de la Escuela de Magisterio de Segovia. REIFOP, 13(3), 27-35. Recuperado de http://aufop.com/aufop/ uploaded_files/articulos/1285861727.pdf

Berrón, E. (2017). Integración creativa de la música Pop-Rock en el aula. Revista electrónica de LEEME, 39, 1-20.

Fraile, A. (2012). Evaluación formativa e interdisciplinariedad. Análisis de dos asignaturas con el mismo sistema de evaluación. Psychology, Society \& Educatión, 4(1),1-12.

Fraile, A., López-Pastor, V. M., Castejón, J., \& Romero, R. (2013). La evaluación formativa en docencia universitaria y el rendimiento académico del alumnado. Aula Abierta, 41(2), 23-34.

Hortigüela, D., Palacios, A., \& López-Pastor, V. M. (2018). The impact of formative and shared or co-assessment on the acquisition of transversal competences in higher education. Assessment \& Evaluation in Higher Education, 43, 933-945. Doi: $10.1080 / 02602938.2018 .1530341$

López-Pastor, V. M. (2010). El papel de la Evaluación Formativa en la evaluación por competencias: aportaciones de la Red de Evaluación Formativa y Compartida en Docencia Universitaria. REDU. Revista de Docencia Universitaria, 9(1), 159173. Recuperado de http://redaberta.usc.es/redu/index.php/REDU

López-Pastor, V. M. (2012). Evaluación formativa y compartida en la Universidad: clarificación de conceptos y propuestas de intervención desde la Red Interuniversitaria de Evaluación Formativa. Psychology, Society \& Education, 4(1), 117-130.

López-Pastor, González, \& Barba (2005). La participación del alumnado en la evaluación: la autoevaluación, la coevaluación y la evaluación compartida. Revista Tándem, 17, 1-7. Recuperado de https://www.researchgate.net/ publication/39211979_La_participacion_del_alumnado_en_la_evaluacion_La_a utoevaluacion_la_coevaluacion_y_la_evaluacion_compartida 
Mitchell, N. (2012). Beginning and Intermediate Piano Students' Experiences Participating in Evaluative Performances. (Tesis Doctoral). Toronto: Universidad de Toronto. Recuperado de https://tspace.library.utoronto.ca/ bitstream/1807/34812/6/Mitchell_Nancy_EC_201211_PhD_thesis.pdf

Romero-Martín, R., Castejón-Oliva, F. J., \& López-Pastor, V. M. (2015). Divergencias del alumnado y del profesorado universitario sobre las dificultades para aplicar la evaluación formativa. Relieve, 21(1), 1-16. Doi:10.7203/relieve.21.1.5169 